\title{
On Spatial Behavior of the Harmonic Vibrations in Kelvin-Voigt Materials
}

\author{
S. Chiriţă • C. Galeş • I.D. Ghiba
}

Received: 26 December 2007 / Published online: 3 June 2008

(C) Springer Science+Business Media B.V. 2008

\begin{abstract}
The present paper deals with the study of the amplitude of the steady-state vibrations in a right finite cylinder made of an isotropic Kelvin-Voigt material. Some exponential decay estimates, similar to those of Saint-Venant type, are obtained for appropriate crosssectional area measures associated with the amplitude of the steady-state vibrations. It is proved that due to dissipative effects, the estimates in question hold for every value of the frequency of vibrations and for arbitrary values of the elastic coefficients. The results are extended to a semi-infinite cylinder and some alternatives of Phragmèn-Lindelöf type are established.
\end{abstract}

Keywords Kelvin-Voigt material $\cdot$ Harmonic vibrations $\cdot$ Spatial behavior $\cdot$ Dissipative effects

Mathematics Subject Classification (2000) 74D05 · 74G50 · 74H45

\section{Introduction}

In the case of viscoelastic materials the distinction between solids and fluids is more or less arbitrary. Solids not only deform but flow to a certain extent and dissipate energy while fluids have a certain amount of rigidity. One of the simplest mathematical models constructed to describe the viscoelastic effects is the classical Kelvin-Voigt model [1, 2]. The basic idea concerning this model is that the stress is dependent on the deformation tensor and deformation-rate tensor. For the one-dimensional linear theory, the Kelvin-Voigt model may be represented by a purely elastic spring and purely viscous damper connected in

S. Chiriţă $(\bowtie) \cdot$ C. Galeş

Faculty of Mathematics, Al. I. Cuza University of Iasi, Blvd. Carol I, no. 11, 700506 Iaşi, Romania e-mail: schirita@uaic.ro

I.D. Ghiba

Octav Mayer Mathematics Institute Iasi, Romanian Academy of Science, Blvd. Carol I, no. 8, 700506 Iaşi, Romania 
parallel. The Kelvin-Voigt material is considered a solid since the deformation is reversible (though not suddenly). More information on the linear theory of viscoelasticity can be found in [3-6].

This paper deals with the three-dimensional linear theory of the Kelvin-Voigt materials (see for example Eringen [3] p. 325 for the basic equations). In fact, we consider a finite (semi-infinite) prismatic cylinder consisting of a homogeneous and isotropic Kelvin-Voigt material, one of whose plane ends is subject to a displacement which varies harmonically in time while the other end (for a finite cylinder) and the lateral surface are clamped, and subject also to standard initial conditions. Following the research line introduced by Flavin and Knops [7] for the linearly damped wave equation and the linearly elastic damped cylinder, and developed further for other models in [8-12] and references therein, we investigate the spatial behavior of solutions describing harmonic vibrations in a Kelvin-Voigt elastic material.

The results obtained in the aforementioned papers suggest exponential decay of activity away from the excited end, provided the frequency of vibration is lower than a critical value and the constitutive coefficients satisfy some positive definiteness conditions. In this work, by using some different measures, we prove that the dissipative effects may be used to obtain the same kind of results but without any restriction condition on the elastic coefficients and for every value of the frequency of vibrations. Of course, the decay rates depend on the Lamé coefficients, the viscosities and the frequency of vibration.

\section{Formulation of the Problem}

Throughout this paper, we refer the motion of a continuum to a fixed system of rectangular Cartesian axes $0 x_{k}(k=1,2,3)$. We shall employ the usual summation and differentiation conventions: Latin subscripts are understood to range over the integers $(1,2,3)$ whereas Greek subscripts are confined to the range $(1,2)$, summation over repeated subscripts is implied, subscripts preceded by a comma denote partial differentiation with respect to the corresponding Cartesian coordinate, and a superposed dot denotes time differentiation.

Let $B$ denote the interior of a right cylinder of length $L>0$ whose cross section is bounded by one or more piecewise smooth curves. Choose the Cartesian coordinates such that the origin lies in one end of the cylinder and such that the $x_{3}$-axis is parallel to the generators. Let $D\left(x_{3}\right)$ denote the cross section of the cylinder corresponding to the axial distance $x_{3}$, and let $\partial D\left(x_{3}\right)$ denote the cross-sectional boundary. We denote by $\pi$ the lateral surface of the cylinder, that is $\pi=\partial D \times[0, L]$.

Let us assume that an isotropic and homogeneous Kelvin-Voigt material occupies $B$. Considering that the natural state is unstressed, then in the absence of the body force, the system of field equations for the linear theory are (cf. Eringen [3], p. 325)

- the equations of motion

$$
t_{r l, r}=\rho_{0} \ddot{u}_{l},
$$

where $\rho_{0}$ is the density mass at time $t=0, t_{r l}$ are the components of the stress tensor, $u_{l}$ are the components of displacement vector field;

- the constitutive equations

$$
t_{r l}=\lambda e_{n n} \delta_{r l}+2 \mu e_{r l}+\lambda^{*} \dot{e}_{n n} \delta_{r l}+2 \mu^{*} \dot{e}_{r l},
$$


where $\delta_{r l}$ is the Kronecker delta, $\lambda$ and $\mu$ are the Lamé coefficients, $\lambda^{*}$ and $\mu^{*}$ are coefficients of viscosity and $e_{r l}$ are defined by

- the geometrical equations

$$
e_{r l}=\frac{1}{2}\left(u_{r, l}+u_{l, r}\right)
$$

The dissipation inequality [3]

$$
\lambda^{*} \dot{e}_{r r} \dot{e}_{l l}+2 \mu^{*} \dot{e}_{r l} \dot{e}_{r l} \geq 0,
$$

implies that

$$
\mu^{*} \geq 0, \quad 3 \lambda^{*}+2 \mu^{*} \geq 0 .
$$

Let us consider the initial boundary value problem defined by the above field equations, the initial conditions

$$
u_{l}=a_{l}^{(1)}\left(x_{1}, x_{2}, x_{3}\right), \quad \dot{u}_{l}=b_{l}^{(1)}\left(x_{1}, x_{2}, x_{3}\right), \quad \text { in } B \text { at } t=0,
$$

and the lateral boundary conditions

$$
u_{l}=0, \quad \text { on } \pi \times\left[0, t_{0}\right)
$$

together with the end boundary conditions

$$
u_{l}=\tilde{u}_{l}\left(x_{1}, x_{2}\right) \exp (-i \omega t), \quad \text { on } D(0) \times\left[0, t_{0}\right)
$$

and

$$
u_{l}=0, \quad \text { on } D(L) \times\left[0, t_{0}\right)
$$

where $\omega$ is a strictly positive constant and $i$ is the unit complex, that is $i=\sqrt{-1}$.

It is easy to see that

$$
u_{l}=U_{l}\left(x_{1}, x_{2}, x_{3}, t\right)+v_{l}\left(x_{1}, x_{2}, x_{3}\right) \exp (-i \omega t)
$$

where $U_{l}$ absorbs the initial conditions and satisfies the null boundary conditions and the equations (2.1), (2.2) and (2.3), while $v_{l}$ satisfies the boundary value problem consisting of the field equations

$$
\begin{aligned}
& T_{r l, r}=-\rho_{0} \omega^{2} v_{l}, \\
& T_{r l}=\left(\lambda-i \omega \lambda^{*}\right) v_{n, n} \delta_{r l}+\left(\mu-i \omega \mu^{*}\right)\left(v_{r, l}+v_{l, r}\right),
\end{aligned}
$$

subject to

$$
v_{l}=0, \quad \text { on } \pi
$$

and

$$
\begin{gathered}
v_{l}=\tilde{u}_{l}\left(x_{1}, x_{2}\right), \quad \text { on } D(0) \\
v_{l}=0, \quad \text { on } D(L) .
\end{gathered}
$$


From (2.11) we deduce the following partial differential equations for the functions $v_{l}$ :

$$
\left(\mu-i \omega \mu^{*}\right) v_{l, r r}+\left[\lambda+\mu-i \omega\left(\lambda^{*}+\mu^{*}\right)\right] v_{r, r l}+\rho_{0} \omega^{2} v_{l}=0 .
$$

It is established in Appendix, under the further assumptions $\mu>0,3 \lambda+2 \mu>0$, that $U_{l}$ tends to zero as $t \rightarrow \infty$. So that, $U_{l}$ represents essentially the transient and $v_{l} \exp (-i \omega t)$ the forced oscillation. We recall that an element of damping has been considered in the equations of motion associated with the problems investigated by Flavin and Knops [7] in order to prove that $U_{l}$ tends to a constant value. In the context of Kelvin-Voigt model these additional terms in the equations of motions are unnecessary because of the dissipative terms present in the constitutive equations.

In what follows we study the spatial behavior of the amplitude of the steady-state vibration satisfying (2.15) under the boundary conditions (2.12), (2.13) and (2.14). Since the effects caused by $U_{l}$ in the cylinder are transient, the decay estimates for $v_{l}$ are of interest for large time $t$.

\section{Spatial Behavior for the Amplitude of the Steady-State Vibration}

In this section we shall study the spatial behavior of the amplitude of the considered vibrations under the assumptions suggested by the dissipation inequality (2.4), that is

$$
\mu^{*}>0, \quad 3 \lambda^{*}+2 \mu^{*} \geq 0 .
$$

To this end we associate with the amplitude of the steady-state vibration the cross-sectional functional

$$
\begin{aligned}
K\left(x_{3}\right)= & -\int_{D\left(x_{3}\right)}\left[i \mu\left(v_{l, 3} \bar{v}_{l}-\bar{v}_{l, 3} v_{l}\right)+i(\lambda+\mu)\left(v_{r, r} \bar{v}_{3}-\bar{v}_{r, r} v_{3}\right)\right. \\
& \left.+\omega \mu^{*}\left(v_{l, 3} \bar{v}_{l}+\bar{v}_{l, 3} v_{l}\right)+\omega\left(\lambda^{*}+\mu^{*}\right)\left(v_{r, r} \bar{v}_{3}+\bar{v}_{r, r} v_{3}\right)\right] d A,
\end{aligned}
$$

where the superposed bar denotes the complex conjugate. Further, we shall establish the following result describing the spatial behavior of the amplitude of the harmonic vibration in concern.

Theorem 1 In the context of a finite cylinder made of a Kelvin-Voigt material, the crosssectional functional $K\left(x_{3}\right)$ represents an acceptable measure of the solution $v_{r}$, in the sense that it is positive for all $v_{r}$ and it vanishes only when $v_{r}=0$. Moreover, it satisfies the following exponential decay estimate

$$
0 \leq K\left(x_{3}\right) \leq K(0) \exp \left(-\frac{2 \omega \mu^{*}}{\xi_{M}} x_{3}\right), \quad x_{3} \in[0, L],
$$

where the positive constant $\xi_{M}$ is given by

$$
\xi_{M}=\frac{1}{\sqrt{\chi_{1}}}\left[|\mu|+4|\lambda+\mu|+\omega\left(4 \lambda^{*}+5 \mu^{*}\right)\right]
$$

and $\chi_{1}$ is the lowest eigenvalue in the two-dimensional clamped membrane problem for the cross section $D$. 
Proof By direct differentiation in (3.2), we obtain

$$
\begin{aligned}
\frac{d K}{d x_{3}}\left(x_{3}\right)= & -\int_{D\left(x_{3}\right)}\left[i(\lambda+\mu)\left(v_{r, r} \bar{v}_{3,3}-\bar{v}_{r, r} v_{3,3}\right)+2 \omega \mu^{*} v_{l, 3} \bar{v}_{l, 3}\right. \\
& \left.+\omega\left(\lambda^{*}+\mu^{*}\right)\left(v_{r, r} \bar{v}_{3,3}+\bar{v}_{r, r} v_{3,3}\right)\right] d A \\
& -\int_{D\left(x_{3}\right)}\left[i \mu\left(v_{l, 33} \bar{v}_{l}-\bar{v}_{l, 33} v_{l}\right)+i(\lambda+\mu)\left(v_{r, r 3} \bar{v}_{3}-\bar{v}_{r, r 3} v_{3}\right)\right. \\
& \left.+\omega \mu^{*}\left(v_{l, 33} \bar{v}_{l}+\bar{v}_{l, 33} v_{l}\right)+\omega\left(\lambda^{*}+\mu^{*}\right)\left(v_{r, r 3} \bar{v}_{3}+\bar{v}_{r, r 3} v_{3}\right)\right] d A .
\end{aligned}
$$

On the basis of (2.15), the square bracket of the second integral may be written in the form

$$
\begin{aligned}
i \mu\left(v_{l, 33} \bar{v}_{l}-\bar{v}_{l, 33} v_{l}\right)+i(\lambda+\mu)\left(v_{r, r 3} \bar{v}_{3}-\bar{v}_{r, r 3} v_{3}\right)+\omega \mu^{*}\left(v_{l, 33} \bar{v}_{l}+\bar{v}_{l, 33} v_{l}\right) \\
\quad+\omega\left(\lambda^{*}+\mu^{*}\right)\left(v_{r, r 3} \bar{v}_{3}+\bar{v}_{r, r 3} v_{3}\right) \\
=-i(\lambda+\mu)\left(v_{r, r \rho} \bar{v}_{\rho}-\bar{v}_{r, r \rho} v_{\rho}\right) \\
\quad-i \mu\left(v_{l, \rho \rho} \bar{v}_{l}-\bar{v}_{l, \rho \rho} v_{l}\right)-\omega \mu^{*}\left(v_{l, \rho \rho} \bar{v}_{l}+\bar{v}_{l, \rho \rho} v_{l}\right) \\
\quad-\omega\left(\lambda^{*}+\mu^{*}\right)\left(v_{r, r \rho} \bar{v}_{\rho}+\bar{v}_{r, r \rho} v_{\rho}\right) .
\end{aligned}
$$

By using the relation (3.6), the divergence theorem and the boundary conditions (2.12) in the second integral of (3.5), we deduce

$$
\frac{d K}{d x_{3}}\left(x_{3}\right)=-\int_{D\left(x_{3}\right)}\left[2 \omega \mu^{*} v_{l, r} \bar{v}_{l, r}+2 \omega\left(\lambda^{*}+\mu^{*}\right) v_{r, r} \bar{v}_{l, l}\right] d A .
$$

Since $\lambda^{*}+\mu^{*}$ is nonnegative, we have

$$
-\frac{d K}{d x_{3}}\left(x_{3}\right) \geq \int_{D\left(x_{3}\right)} 2 \omega \mu^{*} v_{l, r} \bar{v}_{l, r} d A .
$$

On the other hand, by using the arithmetic-geometric inequality, the Schwarz's inequality and

$$
\int_{D\left(x_{3}\right)} v_{l, \rho} \bar{v}_{l, \rho} d A \geq \chi_{1} \int_{D\left(x_{3}\right)} v_{l} \bar{v}_{l} d A,
$$

where $\chi_{1}$ is the lowest eigenvalue in the two-dimensional clamped membrane problem for the cross section $D$, we deduce for the terms in the right hand of (3.2) the following estimates

$$
\begin{aligned}
& \left|\int_{D\left(x_{3}\right)} i \mu\left(v_{l, 3} \bar{v}_{l}-\bar{v}_{l, 3} v_{l}\right) d A\right| \leq 2|\mu|\left(\int_{D\left(x_{3}\right)} v_{l, 3} \bar{v}_{l, 3} d A\right)^{1 / 2}\left(\frac{1}{\chi_{1}} \int_{D\left(x_{3}\right)} v_{l, \rho} \bar{v}_{l, \rho} d A\right)^{1 / 2} \\
& \quad \leq \frac{|\mu|}{\sqrt{\chi_{1}}} \int_{D\left(x_{3}\right)} v_{l, r} \bar{v}_{l, r} d A \\
& \left|\int_{D\left(x_{3}\right)} i(\lambda+\mu)\left(v_{r, r} \bar{v}_{3}-\bar{v}_{r, r} v_{3}\right) d A\right| \leq \frac{|\lambda+\mu|}{\sqrt{\chi_{1}}} \int_{D\left(x_{3}\right)}\left(v_{r, r} \bar{v}_{l, l}+v_{3, \rho} \bar{v}_{3, \rho}\right) d A \\
& \left|\int_{D\left(x_{3}\right)} \omega \mu^{*}\left(v_{l, 3} \bar{v}_{l}+\bar{v}_{l, 3} v_{l}\right) d A\right| \leq \frac{\omega \mu^{*}}{\sqrt{\chi_{1}}} \int_{D\left(x_{3}\right)} v_{l, r} \bar{v}_{l, r} d A
\end{aligned}
$$




$$
\begin{aligned}
& \left|\int_{D\left(x_{3}\right)} \omega\left(\lambda^{*}+\mu^{*}\right)\left(v_{r, r} \bar{v}_{3}+\bar{v}_{r, r} v_{3}\right) d A\right| \\
& \quad \leq \frac{\omega\left(\lambda^{*}+\mu^{*}\right)}{\sqrt{\chi_{1}}} \int_{D\left(x_{3}\right)}\left(v_{r, r} \bar{v}_{l, l}+v_{3, \rho} \bar{v}_{3, \rho}\right) d A .
\end{aligned}
$$

So we can write

$$
\left|K\left(x_{3}\right)\right| \leq \int_{D\left(x_{3}\right)}\left(\mathcal{D}_{1} v_{l, r} \bar{v}_{l, r}+\mathcal{D}_{2} v_{l, l} \bar{v}_{r, r}\right) d A,
$$

where

$$
\begin{aligned}
& \mathcal{D}_{1}=\frac{1}{\sqrt{\chi_{1}}}\left[|\mu|+|\lambda+\mu|+\omega\left(\lambda^{*}+2 \mu^{*}\right)\right], \\
& \mathcal{D}_{2}=\frac{1}{\sqrt{\chi_{1}}}\left[|\lambda+\mu|+\omega\left(\lambda^{*}+\mu^{*}\right)\right] .
\end{aligned}
$$

The distinct eigenvalues of the linear transformation associated with the positive definite quadratic form

$$
\sigma=\mathcal{D}_{1} v_{l, r} \bar{v}_{l, r}+\mathcal{D}_{2} v_{l, l} \bar{v}_{r, r},
$$

are

$$
\xi_{m}=\mathcal{D}_{1}, \quad \xi_{M}=\mathcal{D}_{1}+3 \mathcal{D}_{2} .
$$

From (3.14) we deduce

$$
\left|K\left(x_{3}\right)\right| \leq \int_{D\left(x_{3}\right)} \xi_{M} v_{l, r} \bar{v}_{l, r} d A .
$$

Hence, it follows immediately from (3.8) and (3.18) that

$$
\left|K\left(x_{3}\right)\right| \leq-\frac{\xi_{M}}{2 \omega \mu^{*}} \frac{d K}{d x_{3}}\left(x_{3}\right), \quad x_{3} \in[0, L] .
$$

Since $K(\cdot)$ is a non-increasing function and $K(L)=0$ (see (2.14) and (3.2)), it follows that $K\left(x_{3}\right) \geq 0$ for every $x_{3} \in[0, L]$. Moreover, integrating (3.8) with respect $x_{3}$ on $\left[x_{3}, L\right]$, it follows that

$$
K\left(x_{3}\right) \geq \int_{B\left(x_{3}, L\right)} 2 \omega \mu^{*} v_{l, r} \bar{v}_{l, r} d V,
$$

where $B\left(x_{3}, L\right)=D\left(x_{3}\right) \times\left[x_{3}, L\right]$. This relation together with the boundary conditions prove that $K\left(x_{3}\right)=0$ implies $v_{r}=0$ on $B\left(x_{3}, L\right)$, so that $K\left(x_{3}\right)$ is an acceptable measure of the amplitude of the steady state vibration. Therefore, (3.19) became

$$
\frac{d K}{d x_{3}}\left(x_{3}\right)+\frac{2 \omega \mu^{*}}{\xi_{M}} K\left(x_{3}\right) \leq 0, \quad x_{3} \in[0, L]
$$

By integration one obtains the estimate (3.3) and the proof of Theorem 1 is complete. 
Let us discuss further the case of a semi-infinite cylinder (that is the case when $L \rightarrow \infty$ ). We have the only two possibilities: (a) $K\left(x_{3}\right) \geq 0$ for all $x_{3} \in[0, \infty)$, or (b) there exists $x_{3}^{*} \in[0, \infty)$ so that $K\left(x_{3}^{*}\right)<0$.

Let us first consider case (a). Then the relation (3.19) leads to the differential inequality (3.20) and hence we deduce the spatial decay estimate (3.3). We have to outline that in this case the volume energetic measure

$$
\mathcal{E}\left(x_{3}\right)=\int_{B\left(x_{3}\right)}\left[\mu^{*} v_{l, r} \bar{v}_{l, r}+\left(\lambda^{*}+\mu^{*}\right) v_{r, r} \bar{v}_{l, l}\right] d V,
$$

with $B\left(x_{3}\right)=D\left(x_{3}\right) \times\left[x_{3}, \infty\right)$, exists and is finite.

Let us now discuss case (b). Since $K(\cdot)$ is a non-increasing function on $[0, \infty)$, it follows that we have

$$
K\left(x_{3}\right)<0 \text { for all } x_{3}^{*} \leq x_{3}<\infty
$$

and hence the relation (3.19) leads to the following differential inequality

$$
\frac{d K}{d x_{3}}\left(x_{3}\right)-\frac{2 \omega \mu^{*}}{\xi_{M}} K\left(x_{3}\right) \leq 0, \quad x_{3}^{*} \leq x_{3}<\infty .
$$

By integrating this last differential inequality we obtain the following estimate

$$
-K\left(x_{3}\right) \geq-K\left(x_{3}^{*}\right) \exp \left[\frac{2 \omega \mu^{*}}{\xi_{M}}\left(x_{3}-x_{3}^{*}\right)\right]>0, \quad x_{3}^{*} \leq x_{3}<\infty .
$$

We have to observe that in this case the volume energetic measure $\mathcal{E}\left(x_{3}\right)$ is infinite.

These results are embodied in the following Phragmèn-Lindelöf alternative result.

Theorem 2 In the context of a semi-infinite cylinder we have the following alternative: (a) for the amplitudes having a finite volume energetic measure $\mathcal{E}\left(x_{3}\right)$, the $K\left(x_{3}\right)$, as given by (3.2), is an acceptable measure (in the sense that it is positive for all $v_{r}$ and it vanishes only when $\left.v_{r}=0\right)$ which decays spatially faster than the exponential $\exp \left(-\frac{2 \omega \mu^{*}}{\xi_{M}} x_{3}\right)$, or (b) for the amplitudes having an infinite volume energetic measure $\mathcal{E}\left(x_{3}\right)$, the $-K\left(x_{3}\right)$ grows spatially faster than the exponential $\exp \left[\frac{2 \omega \mu^{*}}{\xi_{M}}\left(x_{3}-x_{3}^{*}\right)\right]$.

\section{Further Measures}

In the present section we prove how we can relax the assumption (3.1), as was suggested by the dissipation inequality (2.4). In fact, we shall establish spatial decay estimates for the relaxed assumptions upon the viscosities $\lambda^{*}$ and $\mu^{*}$ as follows

$$
\mu^{*}>0, \quad \lambda^{*}+2 \mu^{*}>0 .
$$

To this end we introduce the function

$$
\begin{aligned}
J_{\varkappa}\left(x_{3}\right)= & -\int_{D\left(x_{3}\right)}\left[i \mu\left(v_{l, 3} \bar{v}_{l}-\bar{v}_{l, 3} v_{l}\right)+i(\lambda+\mu)\left(v_{r, r} \bar{v}_{3}-\bar{v}_{r, r} v_{3}\right)\right. \\
& +\omega \mu^{*}\left(v_{\rho, 3} \bar{v}_{\rho}+\bar{v}_{\rho, 3} v_{\rho}\right)+\omega\left(\lambda^{*}-\mu^{*}+2 \varkappa\right)\left(v_{\rho, \rho} \bar{v}_{3}+\bar{v}_{\rho, \rho} v_{3}\right) \\
& \left.+\omega\left(\lambda^{*}+2 \mu^{*}\right)\left(v_{3,3} \bar{v}_{3}+\bar{v}_{3,3} v_{3}\right)\right] d A, \quad x_{3} \in[0, L],
\end{aligned}
$$

where $\varkappa$ is a positive parameter at our disposal. Further, we establish the following result. 
Theorem 3 In the context of a finite cylinder, for all $\varkappa \in\left(\max \left(-2 \lambda^{*}-2 \mu^{*}, 0\right), 2 \mu^{*}\right)$, the function $J_{\varkappa}\left(x_{3}\right)$ defined by (4.2) represents a measure of the amplitude of harmonic vibration $v_{r}$, in the sense that it is positive for all $v_{r}$ and it vanishes only when $v_{r}=0$. Moreover, it satisfies the following spatial estimate

$$
0 \leq J_{\varkappa}\left(x_{3}\right) \leq J_{\varkappa}(0) \exp \left(-\frac{2 \omega \sqrt{\chi_{1}}}{\zeta_{M}} x_{3}\right), \quad x_{3} \in[0, L],
$$

where $\zeta_{M}$ is an appropriate positive constant depending on $\lambda, \mu, \lambda^{*}, \mu^{*}, \omega$ and $\varkappa$.

Proof We first note that the direct differentiation and the use of (2.15) and the boundary conditions (2.12) in (4.2) furnish

$$
\begin{aligned}
\frac{d J_{\varkappa}}{d x_{3}}\left(x_{3}\right)= & -2 \omega \int_{D\left(x_{3}\right)} \mu^{*}\left(v_{1,2}-v_{2,1}\right)\left(\bar{v}_{1,2}-\bar{v}_{2,1}\right) d A \\
& -2 \omega \int_{D\left(x_{3}\right)}\left[\left(\lambda^{*}+2 \mu^{*}\right) v_{3,3} \bar{v}_{3,3}+\left(\lambda^{*}+\varkappa\right)\left(v_{\rho, \rho} \bar{v}_{3,3}+\bar{v}_{\rho, \rho} v_{3,3}\right)\right. \\
& \left.+\left(\lambda^{*}+2 \mu^{*}\right) v_{\rho, \rho} \bar{v}_{\alpha, \alpha}\right] d A \\
& -2 \omega \int_{D\left(x_{3}\right)}\left[\mu^{*}\left(v_{3, \alpha} \bar{v}_{3, \alpha}+v_{\alpha, 3} \bar{v}_{\alpha, 3}\right)\right. \\
& \left.+\left(\mu^{*}-\varkappa\right)\left(v_{\rho, 3} \bar{v}_{3, \rho}+\bar{v}_{\rho, 3} v_{3, \rho}\right)\right] d A
\end{aligned}
$$

At this stage we choose the parameter $\varkappa$ in such way that

$$
\max \left(-2 \lambda^{*}-2 \mu^{*}, 0\right)<\varkappa<2 \mu^{*},
$$

so that we have

$$
\begin{aligned}
& \left|\lambda^{*}+\varkappa\right|<\lambda^{*}+2 \mu^{*}, \\
& \left|\mu^{*}-\varkappa\right|<\mu^{*} .
\end{aligned}
$$

With these choices we have

$$
\begin{aligned}
-\frac{d J_{\varkappa}}{d x_{3}}\left(x_{3}\right) \geq & 2 \omega \mu^{*} \int_{D\left(x_{3}\right)}\left(v_{1,2}-v_{2,1}\right)\left(\bar{v}_{1,2}-\bar{v}_{2,1}\right) d A \\
& +2 \omega \tau_{1} \int_{D\left(x_{3}\right)}\left(v_{\rho, \rho} \bar{v}_{\alpha, \alpha}+v_{3,3} \bar{v}_{3,3}\right) d A \\
& +2 \omega \tau_{2} \int_{D\left(x_{3}\right)}\left(v_{3, \alpha} \bar{v}_{3, \alpha}+v_{\alpha, 3} \bar{v}_{\alpha, 3}\right) d A
\end{aligned}
$$

where

$$
\begin{aligned}
& \tau_{1}=\lambda^{*}+2 \mu^{*}-\left|\lambda^{*}+\varkappa\right|>0, \\
& \tau_{2}=\min \left(\varkappa, 2 \mu^{*}-\varkappa\right)>0 .
\end{aligned}
$$

Moreover, we deduce 


$$
\begin{aligned}
-\frac{d J_{\varkappa}}{d x_{3}}\left(x_{3}\right) \geq & 2 \omega \tau_{3} \int_{D\left(x_{3}\right)} v_{\alpha, \rho} \bar{v}_{\alpha, \rho} d A+2 \omega \tau_{1} \int_{D\left(x_{3}\right)} v_{3,3} \bar{v}_{3,3} d A \\
& +2 \omega \tau_{2} \int_{D\left(x_{3}\right)}\left(v_{3, \alpha} \bar{v}_{3, \alpha}+v_{\alpha, 3} \bar{v}_{\alpha, 3}\right) d A
\end{aligned}
$$

where

$$
\tau_{3}=\min \left(\mu^{*}, \tau_{1}\right)
$$

Thus, from (4.10) we deduce that $J_{\varkappa}\left(x_{3}\right)$ is a non-increasing function on $[0, L]$. Since we have $J_{\varkappa}(L)=0$, it follows that $J_{\varkappa}\left(x_{3}\right)$ is a positive quantity and hence, by a procedure similar with that used in theorem 1 , we can conclude that $J_{\varkappa}\left(x_{3}\right)$ appears as an acceptable measure of the amplitude in concern.

Furthermore, we write (4.2) in the form

$$
\begin{aligned}
J_{\varkappa}\left(x_{3}\right)= & -\int_{D\left(x_{3}\right)}\left[i \mu\left(v_{\rho, 3} \bar{v}_{\rho}-\bar{v}_{\rho, 3} v_{\rho}\right)+i(\lambda+\mu)\left(\bar{v}_{\rho} v_{3, \rho}-v_{\rho} \bar{v}_{3, \rho}\right)\right. \\
& \left.+i(\lambda+2 \mu)\left(v_{3,3} \bar{v}_{3}-\bar{v}_{3,3} v_{3}\right)\right] d A-\omega \int_{D\left(x_{3}\right)}\left[\mu^{*}\left(v_{\rho, 3} \bar{v}_{\rho}+\bar{v}_{\rho, 3} v_{\rho}\right)\right. \\
& -\left(\lambda^{*}-\mu^{*}+2 \varkappa\right)\left(\bar{v}_{\rho} v_{3, \rho}+v_{\rho} \bar{v}_{3, \rho}\right) \\
& \left.+\left(\lambda^{*}+2 \mu^{*}\right)\left(v_{3,3} \bar{v}_{3}+\bar{v}_{3,3} v_{3}\right)\right] d A
\end{aligned}
$$

and note that the arithmetic-geometric and Schwarz inequalities and the relation (3.9) give

$$
\begin{aligned}
J_{\varkappa}\left(x_{3}\right) \leq & \frac{1}{\sqrt{\chi_{1}}}\left\{|\mu|+|\lambda+\mu|+\omega\left[\mu^{*}+\left|\lambda^{*}-\mu^{*}+2 \varkappa\right|\right]\right\} \int_{D\left(x_{3}\right)} v_{\alpha, \rho} \bar{v}_{\alpha, \rho} d A \\
& +\frac{1}{\sqrt{\chi_{1}}}\left\{|\lambda+\mu|+|\lambda+2 \mu|+\omega\left[\lambda^{*}+2 \mu^{*}+\left|\lambda^{*}-\mu^{*}+2 \varkappa\right|\right]\right\} \int_{D\left(x_{3}\right)} v_{3, \alpha} \bar{v}_{3, \alpha} d A \\
& +\frac{1}{\sqrt{\chi_{1}}}\left(|\mu|+\omega \mu^{*}\right) \int_{D\left(x_{3}\right)} v_{\rho, 3} \bar{v}_{\rho, 3} d A \\
& +\frac{1}{\sqrt{\chi_{1}}}\left[|\lambda+2 \mu|+\omega\left(\lambda^{*}+2 \mu^{*}\right)\right] \int_{D\left(x_{3}\right)} v_{3,3} \bar{v}_{3,3} d A .
\end{aligned}
$$

From the relations (4.10) and (4.13) we obtain the following first-order differential inequality

$$
2 \omega \sqrt{\chi_{1}} J_{\varkappa}\left(x_{3}\right)+\zeta_{M} \frac{d J_{\varkappa}}{d x_{3}}\left(x_{3}\right) \leq 0, \quad x_{3} \in[0, L],
$$

where

$$
\begin{aligned}
\zeta_{M}=\max \{ & \frac{1}{\tau_{2}}\left(|\mu|+\omega \mu^{*}\right), \frac{1}{\tau_{3}}\left\{|\mu|+|\lambda+\mu|+\omega\left[\mu^{*}+\left|\lambda^{*}-\mu^{*}+2 \varkappa\right|\right]\right\}, \\
& \frac{1}{\tau_{2}}\left\{|\lambda+\mu|+|\lambda+2 \mu|+\omega\left[\lambda^{*}+2 \mu^{*}+\left|\lambda^{*}-\mu^{*}+2 \varkappa\right|\right]\right\}, \\
& \left.\frac{1}{\tau_{1}}\left[|\lambda+2 \mu|+\omega\left(\lambda^{*}+2 \mu^{*}\right)\right]\right\} .
\end{aligned}
$$


Thus, by a direct integration in (4.14) we obtain the decay estimate (4.3) and the proof of theorem is complete.

Following a procedure similar with that in the above section, the results may be easy extended to a semi-infinite cylinder. Thus, we have

Theorem 4 In the context of a semi-infinite cylinder we have the following alternative: (a) for the amplitudes having a finite volume energetic measure $\mathcal{F}\left(x_{3}\right)$, with

$$
\mathcal{F}\left(x_{3}\right)=\int_{B\left(x_{3}\right)} v_{l, r} \bar{v}_{l, r} d V,
$$

the function $J_{\varkappa}\left(x_{3}\right)$, as given by (4.2) with $\varkappa \in\left(\max \left(-2 \lambda^{*}-2 \mu^{*}, 0\right), 2 \mu^{*}\right)$, is a measure which decays spatially faster than the exponential $\exp \left(-\frac{2 \omega \sqrt{x_{1}}}{\zeta_{M}} x_{3}\right)$, or (b) for the amplitudes having an infinite volume energetic measure $\mathcal{F}\left(x_{3}\right)$, the $-J_{\varkappa}^{\zeta}\left(x_{3}\right)$ grows spatially faster than the exponential $\exp \left[\frac{2 \omega \sqrt{\chi_{1}}}{\zeta_{M}}\left(x_{3}-x_{3}^{*}\right)\right]$.

\section{Concluding Remarks}

In this paper we have obtained some spatial decay estimates for the amplitude of harmonic vibration in a right cylindrical region of finite extent, filled by a Kelvin-Voigt elastic material, one plane end of which is subject to an excitation which is harmonic in time. We have emphasized that the dissipative mechanism guarantees the exponential decay of the amplitude of the steady-state vibrations without any restriction upon the elastic coefficients $\lambda$ and $\mu$ and for every value of the frequency of vibrations.

Moreover, if $\mu>0$ and $3 \lambda+2 \mu>0$ then the viscoelastic effects give rise ultimately to a steady state vibration (see the Appendix). So, the whole "activity" decays exponentially from the excited end when $t$ is large enough. When the Lamé coefficients are arbitrary then the same conclusion may be obtained provided some special initial conditions, appropriate to the forced oscillation, are adopted (see [7]).

The results are extended to a semi-infinite cylinder to obtain appropriate alternative of Phragmèn-Lindelöf type.

Acknowledgements The authors are grateful to the reviewers for useful observations leading to improvements of the paper. They also acknowledge support from the Romanian Ministry of Education and Research, CNCSIS Grant code ID-401, Contract no. 15/28.09.2007.

\section{Appendix}

In this section we mimic the algorithm developed by Flavin and Knops [7] in order to prove that $U_{l}$ satisfying

$$
\begin{gathered}
S_{r l, r}=\rho_{0} \ddot{U}_{l}, \\
S_{r l}=\lambda E_{n n} \delta_{r l}+2 \mu E_{r l}+\lambda^{*} \dot{E}_{n n} \delta_{r l}+2 \mu^{*} \dot{E}_{r l}, \quad \text { in } B \\
E_{r l}=\frac{1}{2}\left(U_{r, l}+U_{l, r}\right),
\end{gathered}
$$


subject to boundary conditions

$$
U_{l}=0, \quad \text { on } \partial B
$$

and initial conditions of the type

$$
U_{l}=A_{l}\left(x_{1}, x_{2}, x_{3}\right), \quad \dot{U}_{l}=\dot{A}_{l}\left(x_{1}, x_{2}, x_{3}\right), \quad \text { at } t=0,
$$

decays to zero as $t \rightarrow \infty$. The sufficient conditions assuring this result are $\mu>0$ and $3 \lambda+$ $2 \mu>0$.

By usual procedures, from (A.1) we deduce

$$
\begin{aligned}
& \frac{d}{d t}\left[\int_{B} \frac{\rho_{0}}{2} \dot{U}_{l} \dot{U}_{l} d V+\int_{B}\left(\lambda E_{r r} E_{l l}+2 \mu E_{r l} E_{r l}\right) d V\right] \\
& =-\int_{B}\left(\lambda^{*} \dot{E}_{r r} \dot{E}_{l l}+2 \mu^{*} \dot{E}_{r l} \dot{E}_{r l}\right) d V .
\end{aligned}
$$

Integrating, we deduce that the function

$$
f(t)=\int_{B} \frac{\rho_{0}}{2} \dot{U}_{l} \dot{U}_{l} d V+\int_{B}\left(\lambda E_{r r} E_{l l}+2 \mu E_{r l} E_{r l}\right) d V,
$$

satisfies

$$
f(0)-f(t)=\int_{0}^{t} \int_{B}\left(\lambda^{*} \dot{E}_{r r} \dot{E}_{l l}+2 \mu^{*} \dot{E}_{r l} \dot{E}_{r l}\right) d V d t \geq 0 .
$$

$f(t)$ being non-negative on $[0, \infty)$, we deduce by letting $t \rightarrow \infty$ in the above relation that

$$
\int_{0}^{\infty} \int_{B}\left(\lambda^{*} \dot{E}_{r r} \dot{E}_{l l}+2 \mu^{*} \dot{E}_{r l} \dot{E}_{r l}\right) d V d t<\infty
$$

and moreover, since $\int_{B}\left(\lambda^{*} \dot{E}_{r r} \dot{E}_{l l}+2 \mu^{*} \dot{E}_{r l} \dot{E}_{r l}\right) d V \geq 0$ for all $t$ in $[0, \infty)$, we have

$$
\lim _{t \rightarrow \infty}\left(\lambda^{*} \dot{E}_{r r} \dot{E}_{l l}+2 \mu^{*} \dot{E}_{r l} \dot{E}_{r l}\right)=0, \quad x \in B .
$$

From (3.1), (A.2) and (A.7) it follows that

$$
\lim _{t \rightarrow \infty} \dot{U}_{l}=0
$$

Differentiating (A.1)-(A.3) with respect to $t$ and proceeding as above we obtain

$$
\lim _{t \rightarrow \infty} \ddot{U}_{l}=0
$$

Letting $t \rightarrow \infty$ in (A.1) and (A.2) and taking into account (A.8) and (A.9) we deduce that the limit (as $t \rightarrow \infty$ ) of $U_{l}$, denoted by $U_{l}^{0}$ satisfies

$$
\begin{gathered}
s_{r l, r}=0, \\
s_{r l}=\lambda \varepsilon_{n n} \delta_{r l}+2 \mu \varepsilon_{r l}, \quad \text { in } B \\
\varepsilon_{r l}=\frac{1}{2}\left(U_{r, l}^{0}+U_{l, r}^{0}\right),
\end{gathered}
$$


subject to boundary conditions

$$
U_{l}^{0}=0, \quad \text { on } \partial B .
$$

Since $\mu>0$ and $3 \lambda+2 \mu>0$, the uniqueness result of elastostatics assures that

$$
\lim _{t \rightarrow \infty} U_{l}=U_{l}^{0}=0
$$

\section{References}

1. Voigt, W.: Über die innere Reinbung der festen Körper, insbesondere der Krystalle. Gött. Abh. 36, 1 (1889)

2. Voigt, W.: Über die innere Reinbung der festen Körper, insbesondere der Metalle. Ann. Phys. 47, 671693 (1892)

3. Eringen, A.C.: Mechanics of Continua. Wiley, New York (1967)

4. Leitman, M.J., Fischer, G.M.: The linear theory of viscoelasticity. In: Handbuch der Physik, Band VIa/3. Springer, Berlin (1973)

5. Fabrizio, M., Morro, A.: Mathematical Problems in Linear Viscoelasticity. Society for Industrial and Applied Mathematics, Philadelphia (1992)

6. Christensen, R.M.: Theory of Viscoelasticity: An Introduction, vol. 2. Academic Press, New York (1982)

7. Flavin, J.N., Knops, R.J.: Some spatial decay estimates in continuum dynamics. J. Elast. 17, 249-264 (1987)

8. Flavin, J.N., Knops, R.J., Payne, L.E.: Decay estimates for the constrained elastic cylinder of variable cross section. Q. Appl. Math. 47, 325-350 (1989)

9. Flavin, J.N., Knops, R.J., Payne, L.E.: Energy bounds in dynamical problems for a semi-infinite elastic beam. In: Eason, G., Ogden, R.W. (eds.) Elasticity: Mathematical Methods and Applications. The Ian N. Sneddon 70th Birthday, vol. 1990, pp. 101-112. Ellis Horwood, Chichester (1990)

10. Knops, R.J.: Spatial decay estimates in the vibrating anisotropic elastic beam. In: Rionero, S. (ed.) Waves and Stability in Continuous Media, pp. 192-203. World Scientific, Singapore (1991)

11. Chiriţă, S.: Spatial decay estimates for solutions describing harmonic vibrations in a thermoelastic cylinder. J. Therm. Stress. 18, 421-436 (1995)

12. Aron, M., Chiriţă, S.: Decay and continuous dependence estimates for harmonic vibrations of micropolar elastic cylinders. Arch. Mech. 49, 665-675 (1997) 